\title{
Barriers to Healthcare Access and Long-Term Survival After an Acute Coronary Syndrome
}

\author{
Nathaniel A. Erskine, BA ${ }^{7}$, Molly E. Waring, $P D^{1,2}$, David D. McManus, $M D, \mathrm{MSCl}^{1,3}$, \\ Darleen Lessard, $\mathrm{MS}^{7}$, Catarina I. Kiefe, $\mathrm{PhD}, \mathrm{MD}^{7}$, and Robert J. Goldberg, $P \mathrm{PD}^{7}$
}

'Department of Quantitative Health Sciences, University of Massachusetts Medical School, Worcester, MA, USA; ${ }^{2}$ Department of Allied Health Sciences, College of Agriculture, Health and Natural Resources, University of Connecticut, Storrs, CT, USA; ${ }^{3}$ Division of Cardiovascular Medicine, Department of Medicine, University of Massachusetts Medical School, Worcester, MA, USA.

BACKGROUND: Barriers to healthcare are common in the USA and may result in worse outcomes among hospital survivors of an acute coronary syndrome (ACS).

OBJECTIVE: To examine the relationship between barriers to healthcare and 2-year mortality after hospital discharge for an ACS.

DESIGN: Longitudinal study.

SETTING: Survivors of an ACS hospitalization were recruited from 6 medical centers in central Massachusetts and Georgia in 2011-2013.

PATIENTS: Study participants with a confirmed ACS reported whether they had a financial-related healthcare barrier, no usual source of care, or a transportationrelated healthcare barrier around the time of hospital admission.

\section{INTERVENTIONS: None.}

MEASUREMENTS: Cox regression analyses calculated adjusted hazard ratios (aHRs) for 2-year all-cause mortality for the three healthcare barriers while controlling for several demographic, clinical, and psychosocial characteristics.

RESULTS: The mean age of study participants ( $n=2008)$ was 62 years, 33\% were women, and 77\% were nonHispanic white. One third of patients reported a financial barrier, 17\% lacked a usual source of care, and 12\% had a transportation barrier. Five percent $(n=100)$ died within 2 years after hospital discharge. Compared to patients without these barriers, those lacking a usual source of care and with barriers to transportation experienced significantly higher mortality (aHRs 1.40, 95\% CI 1.30 to 1.51 and $1.46,95 \%$ CI 1.13 to 1.89 , respectively). Financial barriers were not associated with all-cause mortality (aHR 0.79, 95\% CI 0.60 to 1.06).

LIMITATIONS: Observational study with other unmeasured potentially confounding prognostic factors.

CONCLUSIONS: Absence of an established usual source of care and inconsistent transportation availability were associated with a higher risk for dying after an ACS. Patients with these barriers to follow-up care may benefit from more intensive follow-up and support.

Electronic supplementary material The online version of this article (https://doi.org/10.1007/s11606-018-4555-y) contains supplementary material, which is available to authorized users.

Received February 22, 2018

Revised May 25, 2018

Accepted June 11, 2018

Published online July 11, 2018
KEY WORDS: acute coronary syndrome; healthcare barriers; prospective study.

J Gen Intern Med 33(9):1543-50

DOI: $10.1007 / \mathrm{s} 11606-018-4555-\mathrm{y}$

๑) Society of General Internal Medicine 2018

\section{INTRODUCTION}

Medical, physical, and behavioral therapies can reduce mortality among survivors of a hospitalization for an acute coronary syndrome (ACS). ${ }^{1-3}$ These treatments, however, require patients to routinely see clinicians and can cost thousands of dollars in the year following hospital discharge from an ACS. $^{4-6}$ Barriers to healthcare are common among Americans. In 2014, approximately 1 out of every 10 American adults reported lacking health insurance, a similar proportion lacked a usual source of medical care (USOC), and 1 out of 20 were unable to obtain needed care due to its cost. ${ }^{7}$

While current guidelines for the treatment of ACS recommend screening for barriers to healthcare, definitive strategies to address deficits in healthcare access are lacking. ${ }^{1,2} \mathrm{~A}$ limited number of studies have found an inconsistent relationship between different markers of poor access to healthcare, such as lacking a USOC or health insurance, with mortality following hospitalization for an acute myocardial infarction. ${ }^{8-}$

${ }^{12}$ A better understanding of how barriers to healthcare impact mortality following a hospitalization for an ACS could help guide the development of interventions to support disease management post-discharge.

Using data from a prospective cohort study of hospital survivors of an $\mathrm{ACS},{ }^{13}, 14$ we examined the association between financial barriers, no USOC, and transportation barriers to healthcare with 2 year all-cause post-discharge mortality.

\section{METHODS}

\section{Study Sample}

This study uses data from the Transitions, Risks, and Actions in Coronary Events Center for Outcomes Research and Education (TRACE-CORE) investigation. ${ }^{13,14}$ In brief, 2174 adults hospitalized for an ACS were recruited from 3 
community and tertiary care medical centers in Worcester, Massachusetts, 2 hospitals in a managed care network in Atlanta, Georgia, and a tertiary care hospital in Macon, Georgia, between April 2011 and May 2013. Eligible participants were $\geq 21$ years of age and were discharged from participating medical centers after being hospitalized for an ACS. Trained research assistants abstracted data from patient's electronic medical records (EMRs) for the index hospitalization, performed computer-assisted interviews with participants during the index hospitalization or by telephone within $72 \mathrm{~h}$ of discharge, and reviewed state and local vital statistics records to assess patient's mortality status during the 2 years posthospital discharge for their index ACS. The type of data collected by the trained research assistants through the review of EMRs and during the in-person hospital interview is more fully described in the section on potentially confounding variables. The institutional review boards at participating sites approved this longitudinal study.

The analytic sample $(n=2008)$ for the current study consisted of patients with an adjudicated classification of their ACS type and the three measures of healthcare access. We performed multiple imputation by chained equations to estimate the values of potentially confounding covariates for the 204 (10.2\%) participants with missing data. ${ }^{15}$ Patients with missing data were not at greater risk for dying during our 2-year follow-up period than those who had complete data available.

\section{Measuring Barriers to Healthcare Access}

Prior frameworks have identified factors ranging from individual patient attitudes to geography as determinants of healthcare access. ${ }^{16}$ Using the Andersen Model of Access, ${ }^{17,}{ }^{18}$ we examined three enabling or logistical barriers to accessing care in the present study, namely financial barriers, no USOC, and transportation barriers.

\section{Barriers to Healthcare Access}

We considered a financial barrier to healthcare as a lack of insurance coverage and/or self-reported difficulty affording healthcare. We determined insurance status according to EMR documentation. At baseline, interviewers asked participants "in the past twelve months, have you avoided obtaining any health care services because of the cost?" and/or "during the past 12 months, have you had any problems paying medical bills?" ${ }^{10}$ We classified participants who answered yes to either of these questions or who lacked insurance as having a financial barrier.

To determine the presence of a USOC, interviewers asked participants "is there a place that you usually go to when you are sick or need advice about your health?" Interviewers asked participants who responded "yes" to classify the place as a clinic or health center, doctor's office or HMO, emergency room, or outpatient department. ${ }^{7}$ We classified patients who reported no USOC, and those who reported that their USOC was an emergency room, as lacking a USOC. ${ }^{7}$
To assess transportation barriers to healthcare, interviewers asked patients "within the past 12 months, have you missed a medical appointment or been unable to obtain needed health care because of problems with your transportation?" and "overall, and in terms of transportation, how difficult is it for you to get to your health care appointments?" (no problem at all, not very, somewhat, moderately, or extremely difficult). We classified patients who reported missing an appointment in the prior year, or having moderate or extreme difficulty in getting to appointments, as having a transportation barrier.

\section{All-Cause Mortality}

Study coordinators reviewed local and state vital statistics records and hospital EMRs to ascertain patients' survival status during the first 2 years after hospital discharge for their index ACS event.

\section{Potential Confounding Variables}

We examined and analytically controlled for a number of sociodemographic, clinical, and psychosocial characteristics as potential confounders in examining the association between various healthcare barriers and 2-year total mortality.

Demographic Characteristics. Age and sex were abstracted from EMRs by our trained research assistants. During the baseline in-person hospital interview, participants reported their race and ethnicity, household composition, employment status, and level of education. We assessed financial strain by asking patients "in general, how do your finances usually work out at the end of the month?" (some money left over, just enough to make ends meet, not enough to make ends meet). ${ }^{10}$

Clinical Characteristics. Our trained reviewers abstracted data on participants' medical history and hospital course from hospital records after they were discharged from participating hospitals. To confirm and classify patients' type of ACS as STsegment elevation myocardial infarction (STEMI), nonSTEMI (NSTEMI), or unstable angina (UA), two cardiologists reviewed ECG, cardiac biomarker, and cardiac catheterization data from patient's hospital medical records. ${ }^{19,}{ }^{20}$ Patients reported their smoking status (current, former, or never) at baseline. We calculated Global Registry of Acute Coronary Events (GRACE) risk scores (2.0) for mortality using data on clinical characteristics; the GRACE model has demonstrated strong predictive discrimination of mortality for periods of 1 and 3 years after hospital admission for an ACS. ${ }^{21}$

Psychosocial Characteristics. Patients were categorized as having low-health literacy if they reported having little or no confidence when they were asked how confident they were in filling out health forms by themselves during the in-person hospital interview. ${ }^{22}$ To assess patient's cognitive and psychosocial status during their index hospitalization for an ACS, participants completed the 11-item Telephone Interview for Cognitive Status (TICS, range 0 to 41 ); we classified patients 
as being unimpaired ( $\geq 33$ ), ambiguous (26 to 32 ) or moderate to severely impaired $(\leq 25) .{ }^{29}$ We assessed symptoms of depression with the 9-item Patient Health Questionnaire (PHQ9, range 0 to 27) and designated participants as having either no ( $\leq$ 4 ), mild (5 to 9), or moderate to severe ( $\geq 10$ ) depressive symptoms. ${ }^{30}$ We measured symptoms of anxiety with the 7-item Generalized Anxiety Disorder questionnaire (GAD7, range 0 to $21)$ and designated participants as having no $(\leq 4)$, mild (5 to 9$)$, or moderate to severe $(\geq 10)$ symptoms of anxiety. ${ }^{31}$

\section{Data Analysis}

We compared study participants' sociodemographic, clinical, and psychosocial characteristics according to the presence of individual barriers to healthcare access using chi-square tests for categorical variables and unpaired t-tests for continuous variables.

To examine the association of healthcare barriers with allcause mortality in the 2 years after hospital discharge, we calculated unadjusted and multivariable adjusted hazard ratios (HRs) with accompanying 95\% confidence intervals (CIs) using adjusted and unadjusted Cox proportional hazard regression models. ${ }^{23}$ We inspected Schoenfeld residuals and log-log plots $^{23}$ to confirm that the healthcare barriers and additional covariates satisfied the proportional odds assumption.

We first examined the three specific barriers for care (e.g., financial barriers, lack of a USOC, and transportation barriers) in the same regression model. Based on previous research, we decided a priori to include site, sex, race/ethnicity, previously diagnosed diabetes, GRACE risk score, and type of in-hospital reperfusion therapy as controlling variables. We included additional variables that we found that changed the measure of association for one or more of the healthcare barriers with allcause mortality by $10 \%$ or more. We did not include variables already accounted for in the GRACE risk score with the exception of a history of chronic kidney disease. We used a clustered sandwich estimator to calculate the standard errors of coefficients to account for the potential lack of independence of outcomes of patients being treated at the same clinical site. ${ }^{24}$

We performed exploratory analyses of participants according to the eight possible combinations of different healthcare barriers. We constructed Cox proportional hazard models comparing all-cause mortality of study participants with different combinations of healthcare barriers to those with no barriers. Adjusted models included the same variables that we controlled for in the main statistical analysis.

\section{RESULTS}

Among the 2174 TRACE-CORE study participants, we excluded those with missing information on type of ACS $(n=53)$ and any of the three healthcare barriers $(n=113)$, resulting in an analytic sample of 2008. The mean age of this study population was 61.6 years (SD 11.3), 33.1\% were women, and $76.7 \%$ were non-Hispanic white; 15.0, 55.7, and $29.3 \%$ were diagnosed with a STEMI, NSTEMI, and UA, respectively (Table 1).
Approximately one third of study participants reported a financial barrier, $17.1 \%$ lacked a USOC, and $11.9 \%$ reported a transportation barrier; $45.7 \%$ of participants had at least one healthcare barrier. The frequency of these healthcare barriers was $23.6,15.3$, and $8.0 \%$, respectively, among those with a STEMI $(n=301)$ while the frequency of these healthcare barriers was $34.8,17.4$, and $12.6 \%$, respectively, among those with an NSTEMI $(n=1707)$. Participants with each specific healthcare barrier were younger, on average, with smaller proportions reporting to be non-Hispanic white, a college graduate, living with a spouse, retired, or having no monthly financial strain compared to their counterparts who did not have the healthcare barrier of interest (Table 1). Higher proportions of participants with each specific healthcare barrier were currently smoking and had a hospitalization of $\geq 3$ days, but mean GRACE risk scores were lower for those without specific barriers. Low health literacy, mild to severely impaired cognitive status, moderately severe to severe symptoms of depression, and severe symptoms of anxiety were more prevalent among participants with specific healthcare barriers (Table $1 p<0.05$ for all comparisons).

During the 2 years after hospital discharge, $5.0 \%(n=$ $100)$ of all patients died, as did $4.4 \%(n=29)$ of those with a financial barrier, $6.4 \%(n=22)$ of patients without a USOC, and $8.4 \%(n=20)$ of those with a transportation barrier. Those lacking a USOC (adjusted HR 1.40, 95\% CI 1.30 to 1.51 ) and patients with a transportation barrier (adjusted HR 1.46, 95\% CI 1.13 to 1.89) experienced significantly higher 2-year total mortality rates than those without such barriers. Financial barriers were not associated with higher 2-year all-cause mortality (adjusted HR $0.79,95 \%$ CI 0.60 to 1.06 ) (Table 2). These estimates were not materially changed when we further controlled for the receipt of effective hospital medications patients received during their acute hospitalization.

The multivariable adjusted HRs and accompanying 95\% confidence intervals for those with an NSTEMI were 0.90 ( $95 \%$ CI 0.54 to 1.51 ), 1.50 (95\% CI 0.87 to 2.59 ), and 1.44 (95\% CI 0.81 to 2.59 ) for those with a financial barrier, for patients without a USOC, and for patients with a transportation barrier, respectively. We do not present multivariable adjusted HRs for those with a STEMI given the comparatively small number of patients with this type of AMI who were studied, relatively small number of these patients with specific healthcare barriers, and the lack of statistical precision of any adjusted estimates of association between the healthcare barriers of interest and 2-year all-cause death rates.

Eleven percent of study participants reported all three healthcare barriers. Supplement Figure 1 depicts the degree of overlap between different healthcare barriers. Exploratory, multivariable adjusted Cox regressions showed that those with all three barriers and those lacking a USOC with a financial barrier experienced all-cause 2-year mortality rates at either a significantly or non-significantly higher rate than participants reporting no barriers to healthcare (Table 3). 


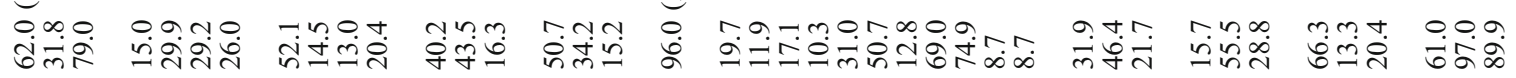

$\hat{\varrho}$

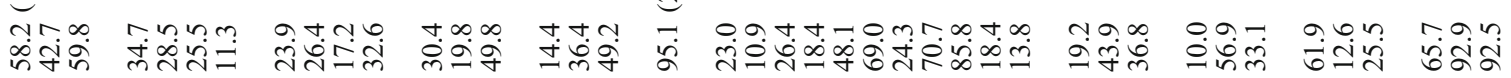

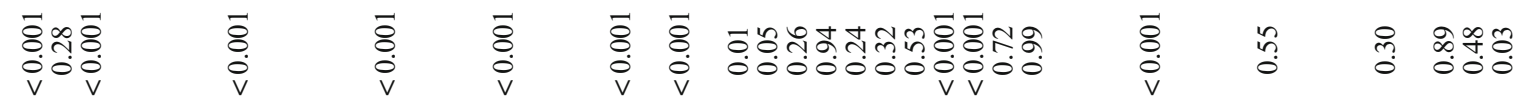

¿

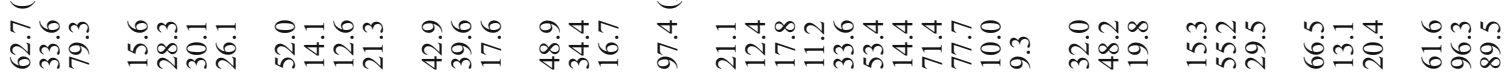

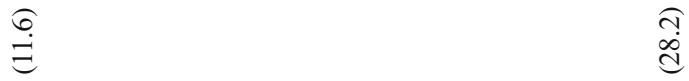

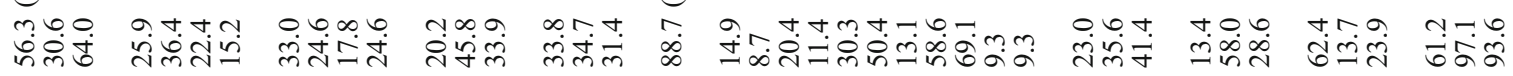

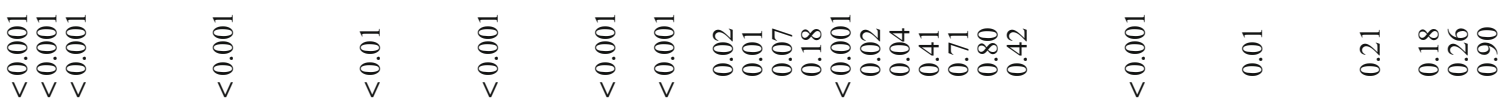

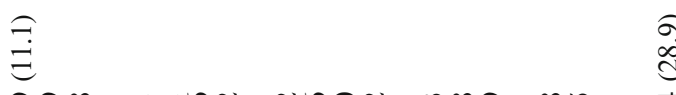

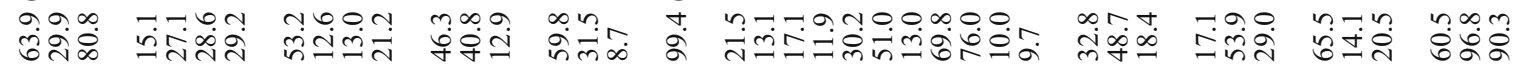

ชิ

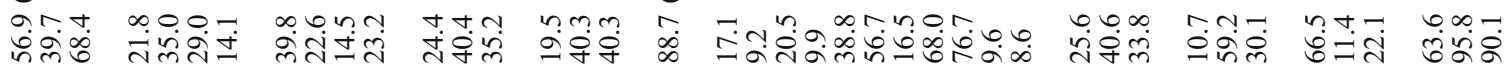
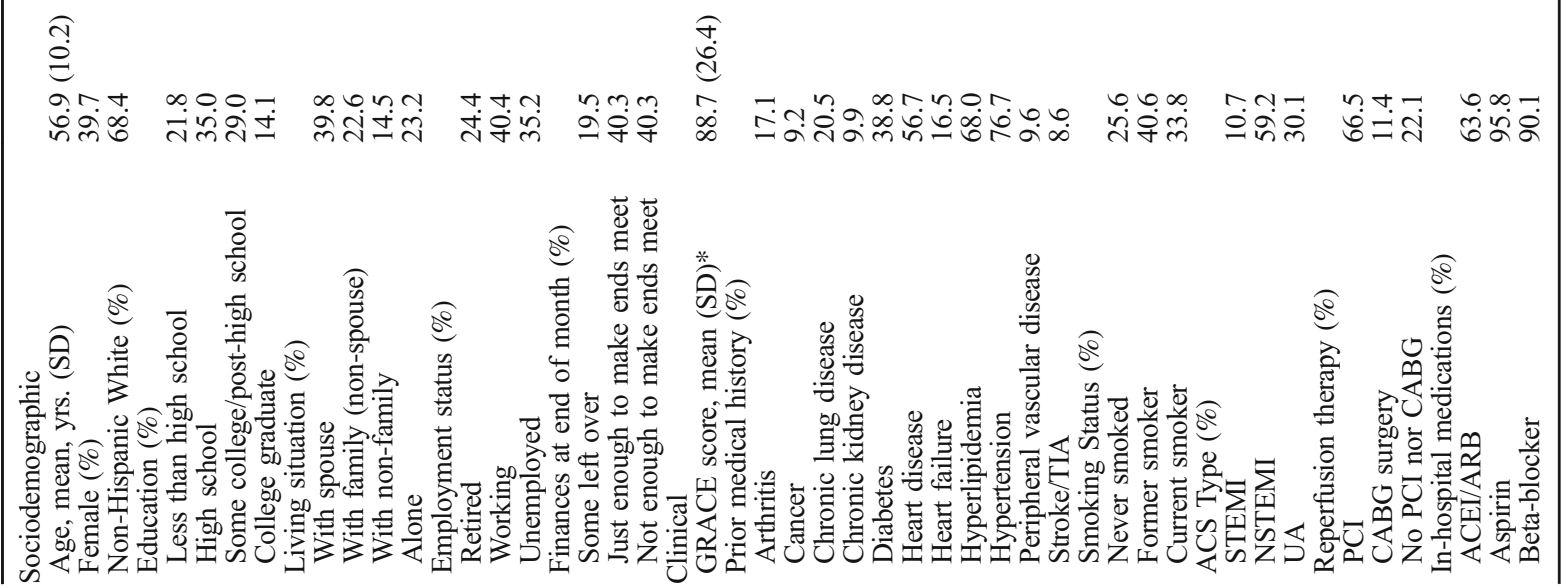


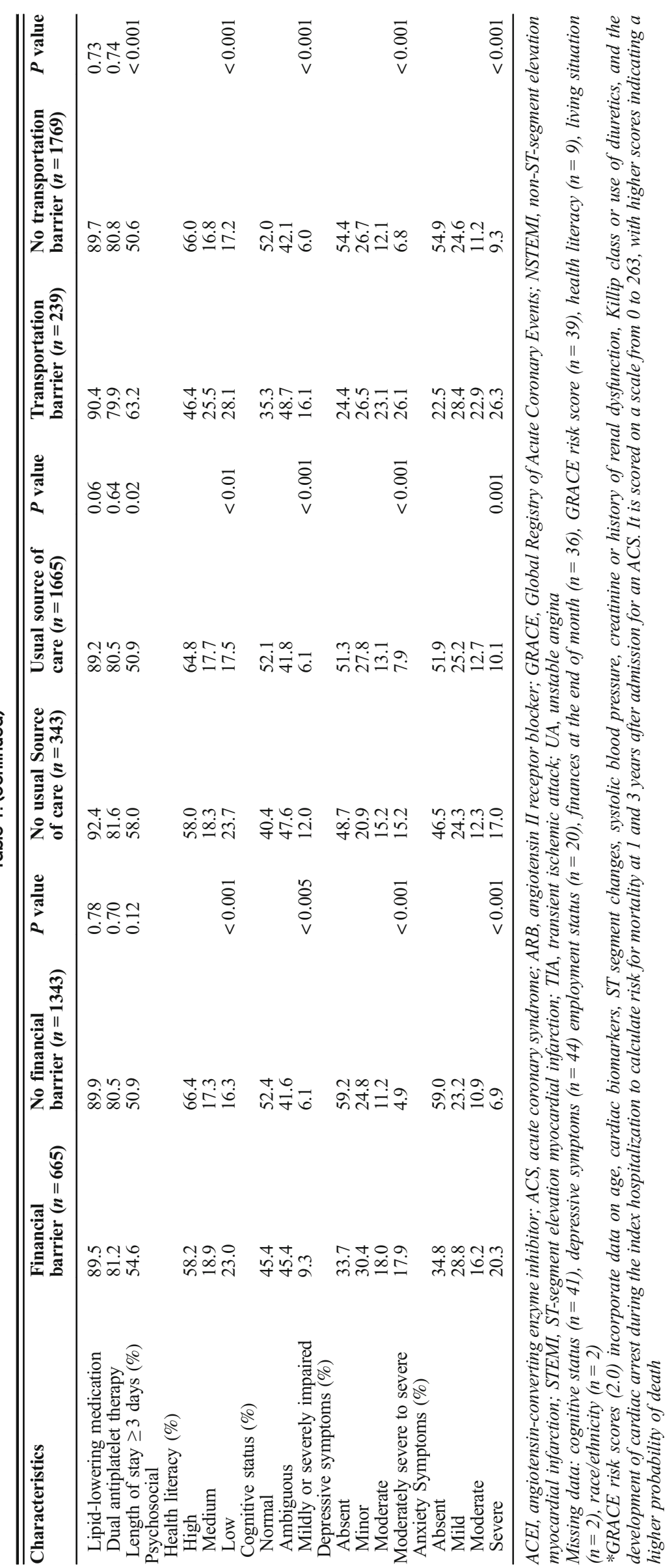


Table 2 Barriers to healthcare access and 2-year all-cause mortality following hospital discharge for an acute coronary syndrome

\begin{tabular}{lll}
\hline \hline Model & Barrier & Hazard ratio (95\% CI) \\
\hline Only adjusted for each specific healthcare barrier & Financial barrier & $0.69(0.40,1.19)$ \\
& No usual source of care & $1.39(1.15,1.68)$ \\
& Transportation barrier & $2.08(1.57,2.74)$ \\
Adjusted for each specific barrier and other potentially & Financial barrier & $0.79(0.60,1.06)$ \\
confounding variables & No usual source of care & $1.40(1.30,1.51)$ \\
& Transportation barrier & $1.46(1.13,1.89)$ \\
\hline
\end{tabular}

Other covariates in the fully adjusted model: site, sex, racelethnicity, GRACE risk score (incorporates data on age, cardiac biomarkers, ST segment changes, systolic blood pressure, creatinine level or history of renal dysfunction, Killip class or use of diuretics, development of cardiac arrest during the index hospitalization), education, living situation, ACS type, hospital receipt of reperfusion therapy, prior heart disease, prior peripheral vascular disease, prior diabetes, depressive symptoms, hospital discharge use of ACE/ARBs, aspirin, beta blockers, lipid lowering agents, and dual antiplatelet therapy

\section{DISCUSSION}

In this prospective study of 2008 hospital survivors of an ACS, almost one half reported barriers related to affording care, having a USOC, and/or transportation. After adjusting for a number and variety of sociodemographic, clinical, and psychosocial characteristics, lacking a USOC and transportation barriers were associated with significantly higher all-cause mortality during the 2 years after hospital discharge. We did not identify an association between financial barriers to care and all-cause mortality. These results highlight that barriers to healthcare access are common among survivors of an ACS and are associated with higher post-discharge death rates, even after accounting for other factors related to a higher risk of dying.

\section{Frequency of Healthcare Barriers}

Our findings of a high prevalence of barriers to healthcare are consistent with results from regional and national studies of hospital survivors of an ACS. The Prospective Registry Evaluating Myocardial Infarction: Event and Recovery (PREMIER) study found that $18 \%$ of 2498 participants reported avoiding healthcare due to costs in the year before hospitalization for an acute myocardial infarction (AMI) at 12 nationwide medical centers between 2003 and $04^{10}$; another analysis of PREMIER participants found that a similar proportion lacked a USOC. ${ }^{8} \mathrm{~A}$ study of 4908 patients hospitalized for AMI at three Maryland hospitals between 1993 and 2008 identified $16 \%$ of patients as being underinsured (i.e., lacking health coverage or having limited coverage). ${ }^{25}$ A qualitative study of 14 patients with heart disease in Canada described how barriers to care can lead patients to miss outpatient appointments and forego medications. ${ }^{26}$ Since access to care may be essential to receiving optimal secondary prevention after an ACS, ${ }^{1,2}$ it may be particularly important for clinicians to routinely screen these patient populations for potential healthcare barriers.

\section{Usual Source of Care}

After accounting for financial barriers to care and transportation access, we found a positive association between lacking a USOC and higher all-cause mortality. An analysis of 2454 survivors of an AMI in the PREMIER study found that those lacking a USOC experienced all-cause death rates at about double the rate of those with an established USOC during the year after hospital discharge. ${ }^{8}$ The presence of a usual source of medical care may confer benefits to patients and the healthcare system by leading to better health screening, ${ }^{27,} 28$ optimal chronic disease treatment, ${ }^{32}$ and reducing the need for emergency care. ${ }^{33,34}$ Lacking a USOC may complicate establishing outpatient follow-up and receiving appropriate secondary prevention medications. Our

Table 3 Exploratory analysis examining combinations of barriers to healthcare access and 2-year all-cause mortality following hospital discharge for an acute coronary syndrome

\begin{tabular}{|c|c|c|c|c|}
\hline Barriers to healthcare access & $\begin{array}{l}\text { Proportion of overall } \\
\text { sample, } n(\%)\end{array}$ & $\begin{array}{l}\text { Two-year } \\
\text { mortality, } n(\%)\end{array}$ & $\begin{array}{l}\text { Crude hazard } \\
\text { ratio (95\% CI) }\end{array}$ & $\begin{array}{l}\text { Adjusted hazard } \\
\text { ratio* }(95 \% \text { CI })\end{array}$ \\
\hline $\begin{array}{l}\text { No healthcare barriers present } \\
1 \text { Barrier present }\end{array}$ & $1090(54.3)$ & $53(4.9)$ & Referent & Referent \\
\hline Financial barrier only & $396(19.7)$ & $12(3.0)$ & $0.61(0.30,1.26)$ & $0.73(0.41,1.30)$ \\
\hline No usual source of care only & $160(8.0)$ & $7(4.4)$ & $0.90(0.80,1.00)$ & $0.92(0.75,1.13)$ \\
\hline Transportation barrier only & $77(3.8)$ & $9(11.7)$ & $2.47(1.06,5.75)$ & $1.80(0.78,4.17)$ \\
\hline \multicolumn{5}{|l|}{2 Barriers present } \\
\hline Financial barrier and no usual source of care & $123(6.1)$ & $8(6.5)$ & $1.37(0.58,3.24)$ & $1.89(1.10,3.26)$ \\
\hline Financial barrier and transportation barrier & $102(5.1)$ & $4(3.9)$ & $0.84(0.28,2.46)$ & $0.71(0.35,1.45)$ \\
\hline No usual source of care and transportation barrier & $16(0.8)$ & $2(12.5)$ & $2.66(0.66,10.73)$ & $2.61(0.89,7.70)$ \\
\hline All 3 healthcare barriers present & $44(2.2)$ & 5 (11.4) & $2.38(2.02,2.81)$ & $1.43(1.19,1.72)$ \\
\hline
\end{tabular}

Adjusted for: site, sex, racelethnicity, GRACE risk score (incorporates data on age, cardiac biomarkers, ST segment changes, systolic blood pressure, creatinine level or history of renal dysfunction, Killip class or use of diuretics, development of cardiac arrest during the index hospitalization), education, living situation, ACS type, hospital receipt of reperfusion therapy, prior heart disease, prior peripheral vascular disease, prior diabetes, depressive symptoms, hospital discharge use of ACE/ARBs, aspirin, beta blockers, lipid-lowering agents, and dual antiplatelet therapy 
exploratory analyses suggested that mortality rates were higher among patients both lacking a USOC and having one or more other barriers to care, but not among those just lacking a USOC. Many individuals may not establish a USOC due to perceptions of good health. ${ }^{35}$ Conceivably, patients lacking only a USOC had good enough health that they chose not to have a USOC, while those possessing a USOC and at least one other healthcare barrier may be more likely to lack a USOC due to an inability to get care. Future studies should further explore the relationship between lacking a USOC with mortality after an ACS, including characterizing the reasons why patients lack a USOC.

\section{Transportation Barriers}

There exists little data on the relationship between barriers to transportation and post-hospital outcomes after an ACS. We identified a higher risk of dying among patients reporting transportation barriers to care. This may be a result of patients being unable to obtain adequate care for the secondary prevention of their heart disease due to transportation related concerns. Our exploratory analyses estimated that those with a transportation related barrier alone had double the mortality as those without any barriers, emphasizing the potential importance of not assuming that a patient has good access because he or she has a USOC and adequate financial resources. A systematic review suggested that lack of transportation is a leading cause of patients missing medical appointments and of the inability to acquire prescription medications. ${ }^{36}$ Despite requirements for many Medicare and Medicaid plans to offer transportation services to patients, many patient transportation services have poor reliability and include excessive wait times. ${ }^{37}$ Further investigation is warranted to assess how ensuring adequate transportation access may improve both short- and long-term clinical outcomes among patients discharged from the hospital after an ACS.

\section{Financial Barriers}

We did not identify an association between financial barriers to healthcare access and utilization with increased all-cause mortality during the 2 years after hospital discharge for an ACS. This may result from patients utilizing emergency services that do not require payment and/or still being able to obtain inexpensive generic medications. ${ }^{38}$ Since our patient recruitment occurred during implementation of the Affordable Care Act, these participants may have subsequently experienced better access due to the expansion of Medicaid, federal subsidies for insurance, and requirements for insurers to cover more health benefits. ${ }^{39}$ However, we could not measure changes in access over the study course. Prior studies have found positive associations of financial barriers to care with higher mortality among those with cardiovascular disease. ${ }^{10,25,40}$ In the aforementioned study of patients hospitalized for an AMI at three Maryland hospitals, the risk of dying from all causes over a 14-year follow-up period was 30\% higher among underinsured patients compared with well-insured patients. ${ }^{25}$ Participants in the PREMIER study who reported avoiding medical care due to cost experienced all-cause mortality within a year of hospital discharge at a $40 \%$ higher rate than for those without such a barrier. ${ }^{10}$ Future studies that track financial status over time may clarify the role of financial barriers and outcomes after an ACS, particularly given the current national debate over the government's role in financing healthcare.

\section{Study Strengths and Limitations}

The strengths of this multi-site prospective study include its large sample of patients with confirmed ACS and ability to investigate multiple types of barriers to healthcare. Nevertheless, we acknowledge several limitations. This study may lack generalizability to patient populations different than those who were hospitalized at participating medical centers. While our multivariable adjusted regression models accounted for known risk factors for mortality post-discharge, including the GRACE risk score and symptoms of depression, ${ }^{21,}{ }^{41}$ there may be other risk factors for all-cause mortality that are more prevalent among patients with no USOC and transportation barriers that are driving the observed findings such as patient's ambulatory status, frailty, and illicit drug or alcohol abuse. We also did not assess the extent of patient compliance to any of the prescribed hospital medications over the course of the 2-year followup nor did we assess whether patients had a follow-up with either a cardiologist or primary care physician during the 4-6 weeks after hospital discharge for their ACS.

\section{CONCLUSIONS}

Hospital survivors of an ACS with barriers to a USOC and transportation exhibited higher all-cause mortality during the 2 years following hospital discharge, suggesting that barriers to healthcare may contribute to poorer long-term health outcomes. Additional research is needed both to confirm our findings in more generalizable patient populations and to explore ways for healthcare systems to best provide services for patients with barriers to care following hospitalization for an ACS.

Acknowledgements: We are indebted to the trained study staff at each of our participating study sites as well as members of our Observational Study Monitoring Board.

Corresponding Author: Robert J. Goldberg, PhD; Department of Quantitative Health Sciences University of Massachusetts Medical School, Worcester, MA, USA (e-mail: robert.goldberg@umassmed.edu).

Funding Sources The National Institutes of Health (NIH) National Heart, Lung, and Blood Institute (NLBI) supported N.E. (1T32HL120823-O1), D.M. (RO1HL126911) and R.G. (1RO1HL135219O1). C.K. received support from the Patient-Centered Outcomes Research Institute (ME-1310-07682) and the NIH National Center for Advancing Translational Sciences (UL1TR0001453-02). The TRACECORE study was funded through NIH NLBI grant UO1HL105268.

\section{Compliance with Ethical Standards:}

Conflict of Interest: The authors of this study have no financial conflicts of interest to disclose. 


\section{REFERENCES}

1. Amsterdam EA, Wenger NK, Brindis RG, Casey DE Jr, Ganiats TG, Holmes DR Jr, et al. 2014 AHA/ACC Guideline for the Management of Patients with Non-ST-Elevation Acute Coronary Syndromes: a report of the American College of Cardiology/American Heart Association Task Force on Practice Guidelines. J Am Coll Cardiol. 2014;64:e139-228.

2. O'Gara PT, Kushner FG, Ascheim DD, Casey DE Jr, Chung MK, de Lemos JA, et al. 2013 ACCF/AHA guideline for the management of STelevation myocardial infarction: a report of the American College of Cardiology Foundation/American Heart Association Task Force on Practice Guidelines. Circulation. 2013;127:e362-425.

3. Smith SC Jr, Benjamin EJ, Bonow RO, Braun LT, Creager MA Franklin BA, et al; World Heart Federation and the Preventive Cardiovascular Nurses Association. AHA/ACCF Secondary Prevention and Risk Reduction Therapy for Patients with Coronary and other Atherosclerotic Vascular Disease: 2011 update: a guideline from the American Heart Association and American College of Cardiology Foundation. Circulation. 2011;124:2458-73.

4. Zhao $\mathbf{Z}$, Winget $\mathbf{M}$. Economic burden of illness of acute coronary syndromes: medical and productivity costs. BMC Health Serv Res. 2011;11:35

5. Simeone JC, Molife C, Marrett E, Frech-Tamas F, Effron MB, Nordstrom BL, et al. One-year post-discharge resource utilization and treatment patterns of patients with acute coronary syndrome managed with percutaneous coronary intervention and treated with ticagrelor or prasugrel. Am J Cardiovasc Drugs. 2015;15:337-50.

6. Mahoney EM, Wang K, Arnold SV, Proskorovsky I, Wiviott S, Antman $\mathbf{E}$, et al. Cost-effectiveness of prasugrel versus clopidogrel in patients with acute coronary syndromes and planned percutaneous coronary intervention: results from the trial to assess improvement in therapeutic outcomes by optimizing platelet inhibition with Prasugrel-Thrombolysis in Myocardial Infarction TRITON-TIMI 38. Circulation. 2010;121:71-9.

7. Ward BW, Clarke TC, Freeman G, Schiller JS. Early release of selected estimates based on data from the 2014 National Health Interview Survey.: National Center for Health Statistics.; 2015

8. Spatz ES, Sheth SD, Gosch KL, Desai MM, Spertus JA, Krumholz HM et al. Usual source of care and outcomes following acute myocardial infarction. J Gen Intern Med. 2014;29:862-9.

9. Gaglia MA Jr, Torguson R, Xue Z, Gonzalez MA, Ben-Dor I, Maluenda G, et al. Effect of insurance type on adverse cardiac events after percutaneous coronary intervention. Am J Cardiol. 2011;107:675-80.

10. Rahimi AR, Spertus JA, Reid KJ, Bernheim SM, Krumholz HM Financial barriers to health care and outcomes after acute myocardial infarction. JAMA. 2007;297:1063-72.

11. Kreindel S, Rosetti R, Goldberg R, Savageau J, Yarzebski J, Gore J, et al. Health insurance coverage and outcome following acute myocardial infarction. A community-wide perspective. Arch Intern Med. $1997 ; 157: 758-62$

12. Beckman AL, Bucholz EM, Zhang W, Xu X, Dryer RP, Strait KM Spertus JA, Krumholz HM, Spatz ES. Sex differences in financial barriers and the relationship to recovery after acute myocardial infarction. J Am Heart Assoc 2016;5:e003923. https://doi.org/10.1161/JAHA. 116.003923.

13. Waring ME, McManus RH, Saczynski JS, Anatchkova MD, McManus DD, Devereaux RS, et al. Transitions, Risks, and Actions in Coronary Events-Center for Outcomes Research and Education (TRACE-CORE): design and rationale. Circ Cardiovasc Qual Outcomes. 2012;5:e44-50.

14. Goldberg RJ, Saczynski JS, McManus DD, Waring ME, McManus R, Allison $\mathbf{J}$, et al. Characteristics of contemporary patients discharged from the hospital after an acute coronary syndrome. Am J Med. 2015; 128:1087-93.

15. White IR, Royston P, Wood AM. Multiple imputation using chained equations: Issues and guidance for practice. Stat Med. 2011;30:377-99.

16. Probst JC, Laditka SB, Wang JY, Johnson AO. Effects of residence and race on burden of travel for care: cross sectional analysis of the 2001 US National Household Travel Survey. BMC Health Serv Res. 2007;7:40.

17. Aday LA, Andersen R. A framework for the study of access to medical care. Health Serv Res. 1974;9:208-20.

18. Andersen RM. Revisiting the behavioral model and access to medical care: does it matter? J Health Soc Behav. 1995;36:1-10.

19. Anderson JL, Adams CD, Antman EM, Bridges CR, Califf RM, Casey DE Jr, et al. 2012 ACCF/AHA focused update incorporated into the ACCF/AHA 2007 guidelines for the management of patients with unstable angina/non-ST-elevation myocardial infarction: a report of the
American College of Cardiology Foundation/American Heart Association Task Force on Practice Guidelines. J Am Coll Cardiol. 2013;61:e179-347.

20. Kushner FG, Hand M, Smith SC Jr, King SB 3rd, Anderson JL, Antman EM, et al. 2009 focused updates: ACC/AHA guidelines for the management of patients with ST-elevation myocardial infarction (updating the 2004 guideline and 2007 focused update) and ACC/AHA/SCAI guidelines on percutaneous coronary intervention (updating the 2005 guideline and 2007 focused update) a report of the American College of Cardiology Foundation/American Heart Association Task Force on Practice Guidelines. J Am Coll Cardiol. 2009;54:2205-41.

21. Fox KA, Fitzgerald G, Puymirat E, Huang W, Carruthers K, Simon T, et al. Should patients with acute coronary disease be stratified for management according to their risk? Derivation, external validation and outcomes using the updated GRACE risk score. BMJ Open. 2014;4:e004425.

22. Powers BJ, Trinh JV, Bosworth HB. Can this patient read and understand written health information? JAMA. 2010;304:76-84.

23. Kleinbaum DG, Klein M. Logistic Regression: A Self-Learning Text. 3rd ed. New York: Springer; 2010.

24. Bragg F, Cromwell DA, Edozien LC, Gurol-Urganci I, Mahmood TA, Templeton A, et al. Variation in rates of caesarean section among English NHS trusts after accounting for maternal and clinical risk: cross sectional study. BMJ. 2010;341:c5065.

25. Ng DK, Brotman DJ, Lau B, Young JH. Insurance status, not race, is associated with mortality after an acute cardiovascular event in Maryland. J Gen Intern Med. 2012;27:1368-1676.

26. Dhaliwal KK, King-Shier K, Manns BJ, Hemmelgarn BR, Stone JA, Campbell DJ. Exploring the impact of financial barriers on secondary prevention of heart disease. BMC Cardiovasc Disord. 2017;17:61.

27. Blewett LA, Johnson PJ, Lee B, Scal PB. When a usual source of care and usual provider matter: adult prevention and screening services. Journal of general internal medicine 2008;23:1354-60.

28. Xu KT. Usual source of care in preventive service use: a regular doctor versus a regular site. Health Serv Res. 2002;37:1509-29.

29. Brandt J, Spencer M, Folstein M. The telephone interview for cognitive status. Neuropsychiatry Neuropsychol Behav Neurol. 1988;1:111-7.

30. Kroenke K, Spitzer RL, Williams JB. The PHQ-9: validity of a brief depression severity measure. J Gen Intern Med. 2001;16:606-13.

31. Spitzer RL, Kroenke $\mathbf{K}$, Williams JB, Lowe B. A brief measure for assessing generalized anxiety disorder: the GAD-7. Arch Intern Med. 2006; 166:1092-7.

32. Spatz ES, Ross JS, Desai MM, Canavan ME, Krumholz HM. Beyond insurance coverage: usual source of care in the treatment of hypertension and hypercholesterolemia. Data from the 2003-2006 National Health and Nutrition Examination Survey. Am Heart J. 2010;160: 115-21.

33. Petterson SM, Rabin D, Phillips RL Jr., Bazemore AW, Dodoo MS. Having a usual source of care reduces ED visits. Am Fam Physician. 2009;79:94.

34. Janke AT, Brody AM, Overbeek DL, Bedford JC, Welch RD, Levy PD. Access to care issues and the role of EDs in the wake of the Affordable Care Act. Am J Emerg Med. 2015;33:181-5.

35. Reasons for Lacking a Usual Source of Care: 2001 Estimates for the US Civilian Noninstitutionalized Population. 2001. at http://www.meps. ahrq.gov/mepsweb/data_files/publications/st32/stat32.pdf.

36. Syed ST, Gerber BS, Sharp LK. Traveling towards disease: transportation barriers to health care access. J Community Health. 2013;38:976-93.

37. Powers BW, Rinefort S, Jain SH. Nonemergency Medical Transportation: Delivering Care in the Era of Lyft and Uber. JAMA. 2016;316:921-2.

38. Carret ML, Fassa AC, Domingues MR. Inappropriate use of emergency services: a systematic review of prevalence and associated factors. Cad Saude Publica. 2009;25:7-28.

39. Blumenthal D, Abrams M, Nuzum R. The Affordable Care Act at 5 Years. N Engl J Med. 2015;372:2451-8.

40. Campbell DJ, Manns BJ, Weaver RG, Hemmelgarn BR, King-Shier KM, Sanmartin C. Financial barriers and adverse clinical outcomes among patients with cardiovascular-related chronic diseases: a cohort study. BMC Med. 2017;15:33.

41. Lichtman JH, Froelicher ES, Blumenthal JA, Carney RM, Doering LV, Frasure-Smith N, Freedland KE, Jaffe AS, Leifheit-Limson EC, Sheps DS, Vaccarino V, Wulsin L. Depression as a risk factor for poor prognosis among patients with acute coronary syndrome: systematic review and recommendations: a scientific statement from the American Heart Association. Circulation. 2014;129:1350-1369. 